\title{
Adaptation des Echinodermes à la vie abyssale
}

\author{
M.SIBUET \\ Centre Océanologique de Bretagne, Brest, France
}

\section{SUMMARY}

The synthesis of literature and personal observations permitted to show the main morphological physiological and genetic characteristics with relation to the mode of life of abyssal echinoderms. The large ventral sole is common among holothuroids and asteroids which creep on the bottom. The development of large podia and their ability to swim are particular to the abyssal family Elasipoda (Holothurioidea). The paper gives examples of opposite skeleton constitution either heavily or lightly calcified. The little knowledge about the biology of these animals shows the absence of a strict diet and of a particular mode of reproduction.

The change of taxonomic composition of the asteroid fauna with depth has also been analysed using the recent synonymy for all available records in the Atlantic Ocean. The relation between the number of families and number of genera related to the number of species unveils, the increase of morphological differences up to a depth range between 2900 and 3200 meters where monospecific genera are rather dominant and about the importance of speciation at greater depths.

\section{INTRODUCTION}

Les conditions de vie considérées souvent comme uniformes dans les grandes profondeurs (nourriture rare, faible température, obscurité, forte pression) ont suscité de nombreuses hypothèses relatives aux caractères adaptatifs de la faune et à $1 \mathrm{a}$ diversité spécifique des peuplements.

Plusieurs écologistes se sont intéressés au rôle sélectif d'un tel milieu et aux possibilités de multiplication des niches écologiques dans un environnement relative- ment uniforme, en particulier Sanders, 1977 ; Grassle et Grassle, 1978. Madsen (1961) et Hansen (1975) ont montré les caractères morphologiques et anatomiques particuliers de familles d'astérides et d'holothurides endémiques des grands fonds dans leurs importantes monographies des Porcellanasteridae et des Elasipoda.

Peu d'êtudes de synthèse ont été menées sur 1 'ensemble d'un embranchement permettant de considérer 1 'éventuelle influence du milieu abyssal et les aspects qui caractérisent la faune vivant par grande profondeur. Les cinq classes d'Echinodermes sont représentées dans les grands fonds et plusieurs ordres et familles sont exclusifs du domaine profond. Afin de présenter les caractéristiques propres des reprësentants abyssaux des différentes classes, plusieurs aspects ont êté retenus :

- les caractéristiques morphologiques, physiologiques et génêtiques des individus vivant au-delà de $2.000 \mathrm{~m}$; ces caractéristiques peuvent être dans certains cas le résultat d'adaptation ou de préadaptation à 1 'environnement abyssal.

- les changements de la composition des différentes classes, en fonction de la profondeur afin de rechercher 1 'importance de la spéciation et des différences rencontrées en zone profonde.

\section{CARACTERISTIQUES MORPHOLOGIQUES - MODE DE VIE}

Un certain nombre de caractères de morphologie externe peuvent être présentés voire corrélés avec ce que l'on sait du mode de vie des échinodermes abyssaux.

\subsection{Les formes typiques de fond dur \\ Ce sont par exemple les crinoídes. Les}


substrats durs (rides océaniques, bordures continentales) sont encore relativement peu explorés et accessibles essentiellement par des moyens photographiques et des observations à partir de submersibles. L'irrégularité de la topographie et les courants variables qui peuvent régner dans les secteurs à substrat rocheux, créent une certaine hétérogénéité du milieu et favorisent 1 'installation des espèces réhophiles à régime alimentaire suspensivore.

\subsection{Les organismes vivant sur les fonds vaseux}

Ces fonds des bassins océaniques sont plus homogènes. Ils peuvent être mieux appréhendés par les moyens de récolte classique. Plusieurs modes de vie existent parmi les échinodermes vivant sur fonds meubles.

\subsubsection{Les formes rampantes}

Ce sont les plus fréquentes. Elles se rencontrent dans les cinq classes et sont caractéristiques du mode de vie de la plupart des espèces densément réparties. Chez ces formes rampantes, on remarque quelques aspects morphologiques particuliers.

- Le développement d'une sole ventrale, large et aplatie se manifeste chez certaines astérides (genre Hymenaster, P1. I.E), certains échinides de grande taille (famille des Echinothuridae), certaines holothuries à symétrie bilatérale (famille des Psychropotidae, Laetmogonidae, et 1 'espèce atlantique Paelopatides gigantea qui vit en populations denses).

- Le développement de podions latéraux est particulier aux holothuries appartenant à l'ordre des Elasipoda qui a une distribution essentiellement bathyale et abyssale. La présence de podions latéraux d'assez grande taille favorise la locomotion chez ces organismes qui laissent des traces en forme de pistes très importantes que $1^{\prime}$ on observe sur les photographies prises près du fond (Hansen, 1972 ; Lemche et al, 1976 ; Pequegnat, 1972).

\section{2 .2 Les formes fouisseuses}

Elles sont relativement rares et représentées par quelques espèces seulement à la distribution clairsemée; les holothuries Molpadidae au corps lisse avec un prolongement caudal, sont les seuls représentants typiquement fouisseurs de cette classe. Les astérides Porcellanasteridae (Pl. I.B) sont supposées être fouisseuses au vu de 1 'existence d'organes cribriformes (Madsen, 1961), et ne sont effectivement jamais observées sur des photos du fond. Les ëchinides irréguliers appartenant au sousordre des Meridosternata qui est exclusivement représenté dans les grands fonds, ont probablement un mode de vie enfoui, mais ces espèces au test particulièrement fragile sont très rares.

\section{2 .3 Les formes nageuses}

Elles sont spécifiques des grands fonds et appartiennent à la plupart des familles de I'ordre des Elasipoda notamment les Psychropotidae et les Elpidiidae. Les espèces de cet ordre confiné au domaine profond ont à la fois la possibilité de nager et de ramper sur le fond. Le développement remarquable de papilles sur la surface du corps de la plupart des espèces de cet ordre assure par ailleurs une augmentation des surfaces d'ëchange ( $\mathrm{Pl}$. I.F).

\subsection{Squelette des échinodermes abyssaux}

La constitution du squelette des échinodermes abyssaux est une autre caractéristique morphologique susceptible d'être marquée par les conditions de vie en $z$ one profonde. En effet, la fixation du calcium est théoriquement plus faible à basse température. De nombreux exemples d'espèces à squelette réduit se rencontrent. Chez les échinides, toutes les espèces de familles essentiellement représentées dans le domaine abyssal ont un test fragile (Meridosternata (P1. I.E) et Echinothuridae). Chez les astérides, deux familles (Brisingidae (P1. I.A), Pterasteridae (P1. I.C)) ayant une distribution essentiellement abyssale, se caractérisent par la fragilité du squelette. Chez les crinoìdes pédonculés, connus en zone profonde, l'allègement du pédoncule et des bras par creusement de 1'areola des articulations a été observé notamment chez les Bathycrinidae (Roux, 1978). S'opposant à ces formes peu calcifiées, des exemples de calcification importante se rencontrent néanmoins. Les Porcellanasteridae ont un squelette développé très rigide (P1. I.B). Des exemples assez contradictoires d'augmentation de la calcification avec la profondeur ont étê mentionnés par Hansen (1975) pour les genres Elpidia et Deima.

3 CARACTERISTIQUES PHYSIOLOGIQUES 
Dans un milieu aux faibles ressources nutritives tel le milieu abyssal, une adaptation vers une physiologie assurant une économie d'énergie est gënéralement supposée. Aucune expérimentation "in situ" par grand fond n'a encore été réalisée sur des échinodermes permettant de mesurer l'intensité du métabolisme ou l'activité enzymatique qui théoriquement diminuent par faible tempërature (Hochachka, 1976). Des mesures "in situ" de consommation d'oxygène réalisées sur des poissons abyssaux sont les seules indications de $1^{\prime}$ existence d'un faible métabolisme en domaine profond (Smith, 1978).

L'étude de la nutrition des échinodermes abyssaux n'a été abordée que rarement à partir de 1 'analyse des contenus digestifs. Sokolova (1972) indique les très grands changements des modes de nutrition en fonction de 1 'augmentation de la profondeur et des changements de substrat, pour l'ensemble de 1 a faune de grande taille. La mégafaune abyssale est essentiellement constituée d'holothurides et d'astérides. Une analyse par Khripounoff (1979) des contenus stomaccaux d'astérides abyssales récoltées dans le golfe de Gascogne apporte une dëmonstration de ces changements déjà suggérés par Carey (1972) chez des astérides du Pacifique. La proportion importante d'astérides carnivores aux faibles profondeurs diminue pour des profondeurs croissantes et est remplacée dans les grands fonds par des espèces limivores et omnivores. Dans les autres classes d'échinodermes, les espèces vivant dans les grands fonds sont également opportunistes et mangent du sédiment, à l'exception des crinoïdes suspensivores, plus rares sur les fonds vaseux. Un fait est particulièrement frappant chez les holothuries dont le mode de vie est essentiellement rampant, leur tube digestif est presque toujours rempli de vase; ceci peut suggérer une ingestion continue permettant un apport organique suffisant.

Les capacités de sélection de la nourriture peuvent être une autre possibilité de se maintenir dans un milieu pauvre mais ne sont toutefois pas spécifiques des grands fonds. La mise en évidence de la sêlection d'un sêdiment plus riche en matière organique a été faite chez certaines holothuries abyssales de 1 'ordre des Elasipoda (Sokolova, 1958 ; Bordovskiy et a1, 1974 ; Khripounoff, 1979). Mais la manifestation d'une sélection de la nourriture ne peut être attribuée à l'ensemble des holothurides abyssales. Chez les genres Molpadia et Pseudostichopus répandus dans les grands fonds, 1'absence de sélection a été notée (Sokolova, 1958; Khripounoff, 1979). Devant la raréfaction de la nourriture avec l'augmentation de la profondeur et l'éloi- gnement des continents, une question peut se poser concernant la constitution biochimique des tissus. Des indications de faibles teneurs en matières organiques ont été mentionnées par Hansen (1975) pour le genre Elpidia et par Barnes et al (1976) pour Peniagone diaphana. Mais des analyses effectuées sur les principales holothuries abyssales du golfe de Gascogne démontrent que les proportions en protéines, lipides et glucides dans les téguments sont les mêmes que pour des espèces littorales (Lawrence et Sibuet, 1979).

Les modes et cycles éventuels de reproduction sont très mal connus chez les espèces abyssales. Les analyses de structure de population et de variation de l'indice gonadique qui indiquent, pour quelques espèces d'ophiures eurybathes, 1 'existence de cycle périodique de reproduction $n^{\prime}$ ont pu être réalisées que sur des populations abondantes (Schoener, 1968 ; Rokop, 1975 ; Tyler et Gage, 1980). Ces méthodes ne permettent pas l'étude des espèces typiquement abyssales vivant en populations souvent dispersées. Comme la dispersion entre les individus d'une même espèce est très grande chez la plupart des échinodermes abyssaux, on peut se demander quels sont les facteurs qui favorisent la fertilisation. Hansen (1968) a signalé chez Oneirophanta mutabilis le cas d'un embryon intraovarien se nourrissant d'oeufs non fécondés. De nombreuses observations de 1 'augmentation de la taille des oeufs chez certaines espèces d'holothurides (Hansen, 1975), d'astérides (Madsen, 1961) d'ophiures (Schoener, 1968) peuvent faire penser à une possibilité soit de réduction du stade larvaire pélagique, soit de l'existence d'un stock de réserves pour la survie larvaire dans un milieu appauvri.

\section{CARACTERISTIQUES GENETIQUES}

La variabilité génétique peut rendre compte de l'adaptation ou de la pression sélective du milieu abyssal et intervenir sur 1a distribution des espèces. D'après Mayr (1974) une variabilité génétique élevée favorise une plus ample utilisation du milieu car elle permet la colonisation d'habitats marginaux et de sous-niches diverses. D'après Grassle (1972) et Grassle et Grassle (1978) une faible variabilité génétique serait rencontrée chez les espèces adaptées à un milieu où sévit une forte pression sélective. Plusieurs auteurs, notamment Murphy et a1 (1977) et Costa et Bisol (1978) ont cherché à traduire la variabilité génétique par le degré de polymorphisme de différents enzymes, et à corréler le taux de polymorphisme et la 
stabilité du milieu abyssal. Chez les espèces d'échinodermes étudiées par ces auteurs, le polymorphisme est élevé pour des espèces eurybathes et cosmopolites telles Ophiomusium lymani (P1. I.D). Aucune espèce à distribution strictement abyssale n'a encore fait 1 'objet de telles analyses.

5 COMPOSITION DES DIFFERENTES CLASSES D'ECHINODERMES EN FONCTION DE LA PROFONDEUR

En l'absence de recensement de la faune échinodermique des différents océans, 1 'analyse des variations de la composition specifique en fonction de 1 a profondeur ne peut être que partielle.

Les données d'Ekman (1954) permettent de montrer à l'échelle de l'ocëan Atlantique, une diminution progressive du nombre d'espèces suivant des profondeurs croissantes, pour les trois classes des astérides, échinides, ophiurides.

REPARTITION DES ECHINODERMES ATLANTIOUES EN FONCTION DE LA PROFONDEUR

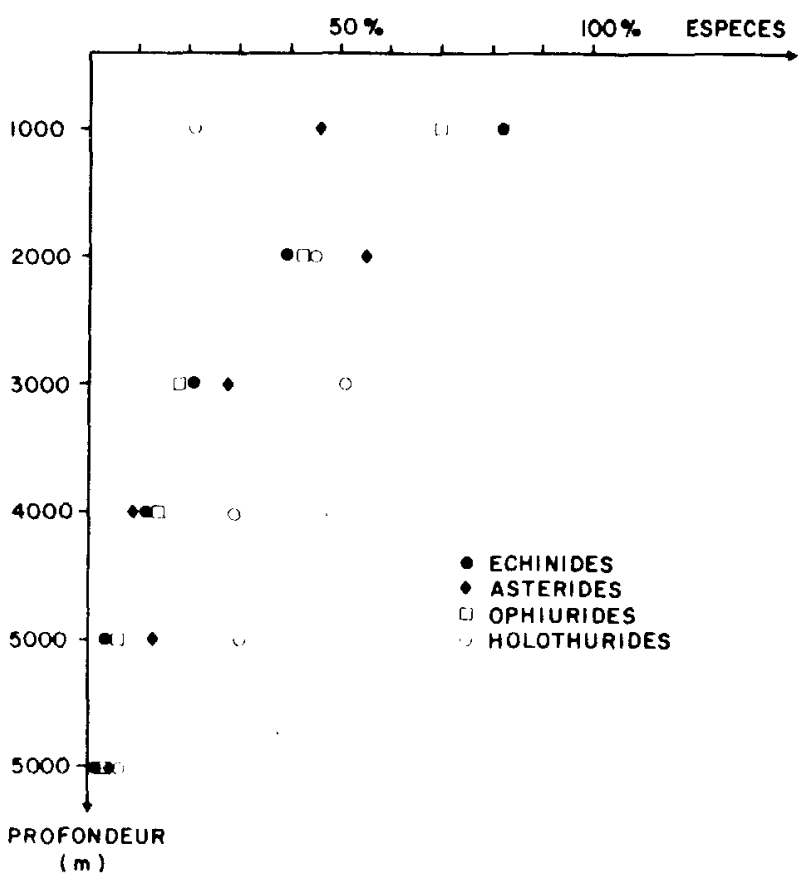

Figure 1. Répartition en terme de pourcentages de quatre classes d'échinodermes en domaine profond dans l'océan Atlantique (d'après les données d'Ekman, 1954).

La classe des holothurides se distingue par un accroissement du nombre d'espèces vers des profondeurs de $3.000 \mathrm{~m}$. Si ces résultats peuvent être en partie faussés par un échantillonnage incomplet dans différentes zones de profondeurs, il est intéressant de les comparer avec les résultats obtenus dans un secteur géographique restreint et suffisamment échantillonné comme le golfe de Gascogne (Laubier et Sibuet, 1977,1979). La figure 2 est réalisée à partir des résultats de l'étude des échinodermes récoltês intensivement dans le golfe de Gascogne. La même différence de richesse spécifique est observée pour les 4 classes étudiēes, aux différentes profondeurs.

Le succès des holothurides vers les profondeurs de $3.000 \mathrm{~m}$ montre que l'environnement abyssal a pu être favorable à la spéciation de cette classe. Les holothuries présentent en effet un meilleur rayonnement adaptatif que les autres classes dans les régions profondes. Les résultats concernant la diversité spécifique des holothurides et des astérides (Sibuet, 1977) ont montré 1 'existence d'une diversité faible dans les grandes profondeurs à I'exception des holothurides dont l'indice de diversité est plus élevé vers $3.000 \mathrm{~m}$. Ces résultats s'opposent, en partie, à l'hypothèse de Sanders (1969), qui suggère qu'un environnement stable favorise une diversité spécifique élevée. Grassle et Grassle (1978) cherchent une explication à cette hypothèse, et pensent que la diversification de niches trophiques peut être plus importante en zone profonde pour certains groupes zoologiques (Polychètes, Bivalves, ...). L'embranchement des échinodermes, qui est caractérise dans les grands fonds par des espèces ayant de grandes aires de répartition géographique, ne répond pas aux mêmes exigences. La figure 3 donne une représentation du nombre de genres et de familles pour chaque classe d'échinodermes vivant par

NOMBRE D'ESPECES D'ECHINODERMES EN FONCTION DE LA PROFONDEUR DANS LE GOLFE DE GASCOGNE

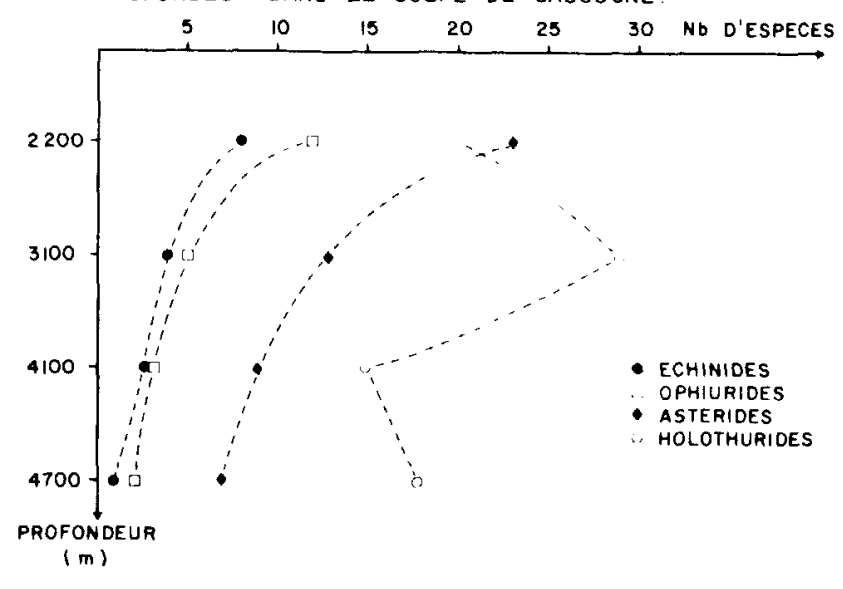

Figure 2. Répartition du nombre d'espèces de quatre classes d'échinodermes dans le golfe de Gascogne. 


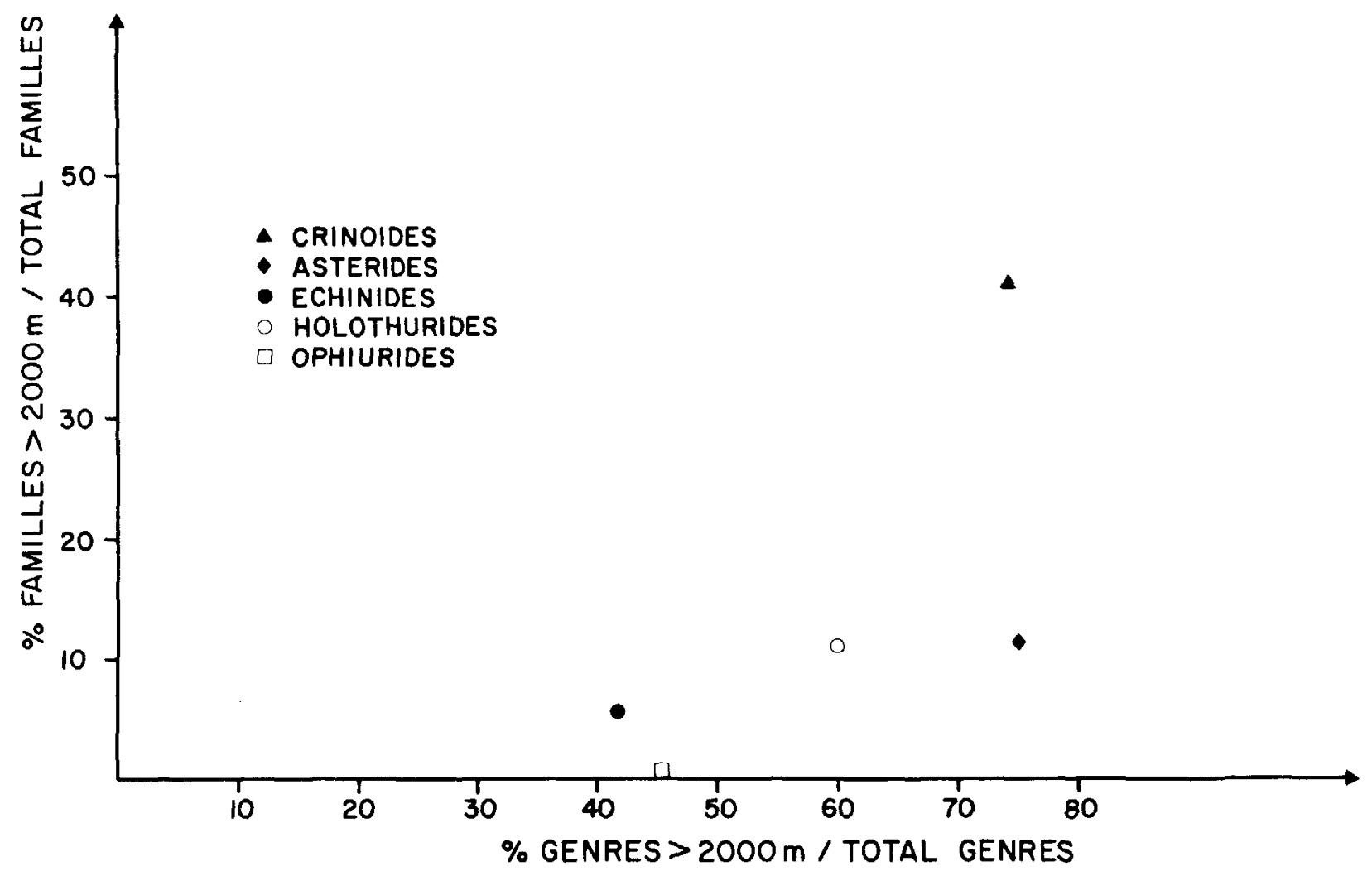

Figure 3. Représentation en terme de pourcentage par rapport au total connu des familles et genres existant à des profondeurs supérieures à $2.000 \mathrm{~m}$ mais inexistant à des profondeurs inférieures à $500 \mathrm{~m}$. (d'après les données de Birstein et Vinogradov 1971).

plus de $2.000 \mathrm{~m}$ et absent dans les fonds inférieurs à $500 \mathrm{~m}$ (à. partir des données recueillies par Birstein et Vinogradov (1971). La classification, bien que souvent arbitraire, au niveau d'un genre ou d'une famille indique toutefois I'importance des diffërences morphologiques entre les espèces. La proportion assez importante de genres de crinoïdes, d'astérides et d'holothurides (figure 3) dans le domaine profond par rapport à la totalité des genres connus, montre 1 'importance de la différenciation morphologique dans ce milieu.

Une caractérisation des espèces d'astérides représentées dans différentes tranches de profondeurs a été recherchée à partir du recensement de la totalité des espèces d'astérides rêcoltêes dans 1 'océan At lantique (figure 4). Ces astérides, recensées et archivées au Centre Océanologique de Bretagne, ont été classées selon la synonymie actuelle (communication personne1le de A.M. Clark).

Le classement des espèces a été ordonné pour suivre les variations des rapports, nombre de genres/nombre d'espèces, en fonction de 1 'accroissement en profondeur avec un intervalle de $300 \mathrm{~m}$ retenu arbitrairement. L'analyse porte sur les va- riations du nombre de genres et de familles par rapport au nombre d'espèces, ce qui permet d'apprécier les différences morphologiques entre les espèces dans ces tranches de profondeurs distinctes. L'ensemble des points n'est analysable que si 1 'on opère des regroupements en 4 nuages en tenant compte de la bathymêtrie. Les points du nuage I (correspondant aux espèces vivant entre 200 et $2300 \mathrm{~m}$ ) ne présentent pas de corrélation particulière. Les points 8 et 9 du nuage II (entre 2300 et $2900 \mathrm{~m})$ se distinguent par une nette tendance à 1 'accroissement du nombre de genres par rapport au nombre d'espèces. Le point 10 (entre 2900 et $3200 \mathrm{~m}$ ) présente les valeurs maximales des rapports genres/ espèces et familles/espèces. Le nombre de genres très proche du nombre d'espèces est la conséquence de la fréquence élevée de genres monospécifiques. Il est remarquable de noter que les points de 10 à 14 (bathymétrie supérieure à $2900 \mathrm{~m}$ ) s'alignent selon une droite, ce qui indique une nette corrélation entre les valeurs des rapports genres/espèces et familles/espèces. Ces points sont ordonnés selon une profondeur croissante du point 10 au point 13 (de 2900 à $4100 \mathrm{~m}$ ). Le point 14 


\section{ASTERIDES ATLANTIQUES}

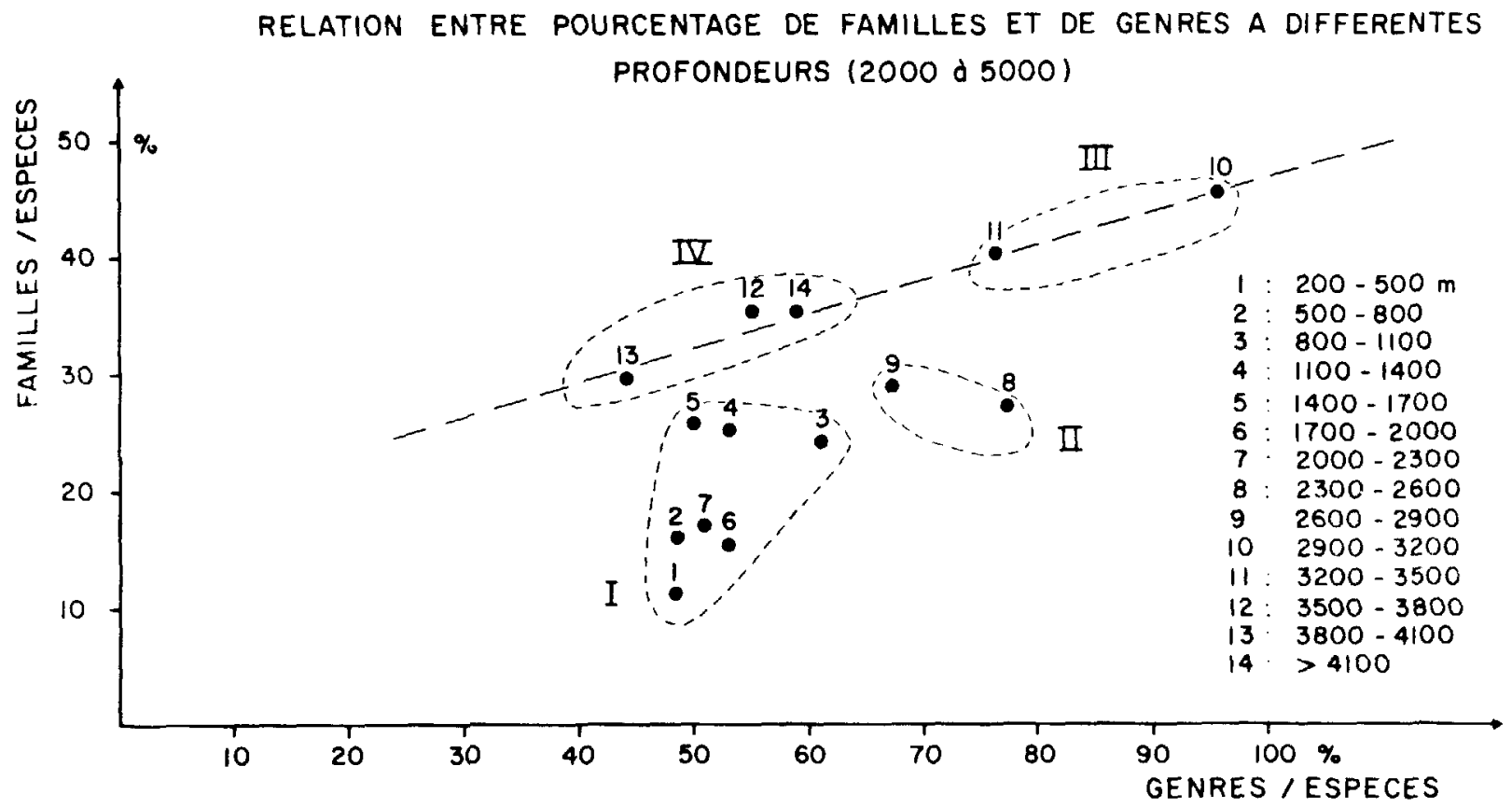

Figure 4. Représentation en terme de pourcentages des genres et familles par rapport aux espèces présentes à diffërentes tranches de profondeurs dans l'océan Atlantique.

(supërieur à $4100 \mathrm{~m}$ ) présentant une valeur proche du point 12 , les points 12 à 14 ont été regroupés dans le nuage IV (plus de $3,(10 \mathrm{~m})$; les points 10 et 11 constituent 1t. nuage III $(2900$ à $3500 \mathrm{~m})$. La position des nuages les uns par rapport aux autres montre qu'entre $2900 \mathrm{~m}$ et $3500 \mathrm{~m}$ les taxons de rang supérieur ne sont représentés que par un faible nombre d'espèces voire par une seule. Les nuages I et II d'une part et le nuage IV d'autre part se distinguent par les valeurs du rapport familles/ espèces, celui-ci est systématiquement plus élevé pour les grandes profondeurs et tra-

\section{LEGENDE DE LA PLANCHE I}

Photographies d'échinodermes récoltês, puis placés en aquarium au cours des campagnes organisées par le Centre Océanologique de Bretagne.

A. Astéride, Brisingidae, Freyella spinosa E. Perrier $(G \times 0,35)$, lieu de récolte : Golfe de Gascogne, $47^{\circ} 33,2 \mathrm{~N} 8^{\circ} 41,4 \mathrm{~W}$, $2177 \mathrm{~m}$.

B. Astéride, Porcellanasteridae, Styracaster chuni Ludwig $(G \times 0,60)$, lieu de récolte: Golfe de Gascogne, $46^{\circ} 30,6 \mathrm{~N} 10^{\circ} 30 \mathrm{~W}$, $4721 \mathrm{~m}$.

C. Astéride, Pterasteridae, Hymenaster Pellucidus Wyv. Thomson $(G \times 1)$, 1 ieu de duit des différences morphologiques plus importantes.

L'ordre de ces nuages permet de constater que jusque vers $2900 \mathrm{~m}$ et $3200 \mathrm{~m}$ le taux de spéciation des astérides semble décroître puis augmenter de nouveau au-delà de $3200 \mathrm{~m}$; les valeurs du nuage IV, pour un nombre rëduit d'espèces, convergent en effet vers celles du nuage $I$. Ces résultats montrent que les astérides sont relativement bien adaptées aux grandes profondeurs. Cette analyse globale à l'échelle de 1 'At lantique montre que les différences entre les taxons tendent à augmenter selon

récolte : Mer de Norvège, $64^{\circ} 26,5 \mathrm{~N}$ $01^{\circ} 36,1 \mathrm{~W}, 2714 \mathrm{~m}$.

D. Ophiuride, Ophiolepidae, Ophiomusium lymani Wyv. Thomson $(G \times 0,4)$, lieu de récolte : Golfe he Gascogne, $47^{\circ} 33,5 \mathrm{~N}$ $8^{\circ} 45 \mathrm{~W}, 2350 \mathrm{~m}$.

E. Echinide, Pourtalesiidae, Pourtalesia jeffreysi Wyv. Thomson $(G \times 1,5)$, lieu de récolte : Mer de Norvëge, $73^{\circ} 28,2 \mathrm{~N}$ $10^{\circ} \mathrm{O6}, 6 \mathrm{~W}, 2937 \mathrm{~m}$.

F. Holothuride, Elasipodidae, Psychropotes depressa (Thee1) ( $G \times 0,5)$, lieu de récolte : Golfe de Gascogne, $47^{\circ} 30,9 \mathrm{~N}$ $8^{\circ} 59,5 \mathrm{~W}, 2695 \mathrm{~m}$.

$\mathrm{N}^{\circ}$ de contribution du Département Etudes Océaniques/C.0.B. 672. 

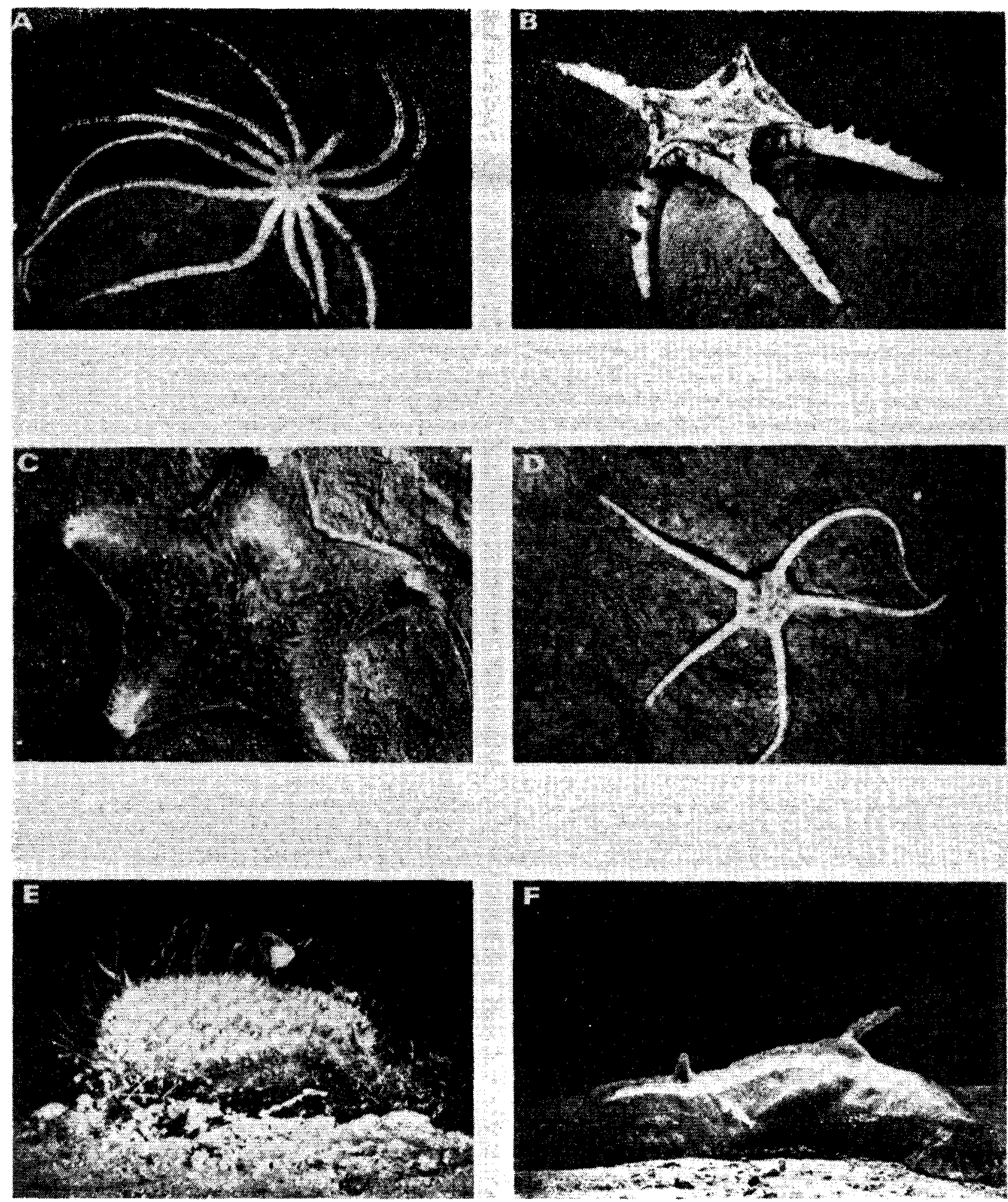
des profondeurs croissantes jusqu'à une certaine gamme de profondeur située aux environs de $3.000 \mathrm{~m}$. Ces premiers résultats d'une analyse globale nécessitent d'être mieux approfondis à partir d'études plus localisées afin de rendre compte de 1 'influence d'autres paramètres écologiques susceptibles de varier suivant les secteurs géographiques.

\section{n CONCLUSION}

'étude des caractéristiques individuelles its échinodermes abyssaux montre, à partir de données toutefois encore fragmentaires, qu'un mode de comportement rampant domine hiez toutes les classes. Ceci se remarque dans le développement de la sole ventrale et 1 'importance des podions notamment chez les holothuries Elasipoda confinées dans les grands fonds. L'association chez certaines holothuries Elasipoda de ce mode de comportement avec la nage est une autre particularité. La constitution du squelette apparaît relativement allégëe pour l'ensemble des espèces mais des exemples contradictoires empêchent d'établir une règle générale de diminution de la calcification pour des profondeurs croissantes. Les quelques observations d'ordre physiologique très rarement menées sur des espèces strictement abyssales, permettent de constater $I^{\prime}$ absence de régime alimentaire strict et $1^{\prime}$ absence d'un mode de reproduction bien particu1ier. De grandes tolérances physiologiques semblent exister chez des espèces pour la plupart eurybathes et cosmopolites, mais les mécanismes qui interviennent dans la survie de populations souvent très dispersêes dans les zones profondes ne peuvent être expliqués.

Les holothurides et astérides apparaissent comme les classes les mieux représentées dans les grandes profondeurs. Vers $3.000 \mathrm{~m}$, les holothurides présentent leur maximum de diversité spécifique. Des changements dans la composition taxonomique des astérides de l'Atlantique, en fonction de la profondeur, apparaissent à travers la distribution des genres et familles par rapport aux espèces semble mettre en évidence : une augmentation des différences morphologiques vers $2900-3200 \mathrm{~m}$, où une tendance aux genres monospécifiques est nettement marquée, et une spéciation relativement importante aux grandes profondeurs.

\section{REME RC IEMENTS}

J'exprime ma reconnaissance à

D. Desbruyères, L. Laubier, C. Massin,
D. Reyss et M. Roux pour leurs suggestions lors de la lecture du manuscrit.

\section{REFERENCES}

Barnes, A.T., L.B. Quetin, J.J. Childress \& D.L. Pawson 1976, Deep sea macroplanktonj.c sea cucumbers : suspended sediment feeders cantured from deep submergence vehicle, Science 194:1083-1085.

Birstein, J.A. \& M.E. Vinogradov 1971, Role of the trophic factor in the taxonomic discreteness of marine deep sea fauna, Bul1.Soc. Natural. Moscou, Biol.76(3): 59-92 (en russe).

Bordovskiy, O.K., M.N. Sokolova, B.A. Smirnov, Y.A. Akhmet'yeva \& O.N. Zezina 1974, Evaluation of the role of bottom fauna in the transformation of organic matter in sediments (with specific reference to the deep sea detritus), Oceanology 14:128-132.

Carey Jr, A.G. 1972, Food sources of sublittoral, bathyal and abyssal asteroids in the northeast Pacific ocean, Ophelia, 10:35-47.

Costa, R. \& P.M. Bisol 1978, Genetic variability in deep sea organisms, Biol.Bull. 155:125-133.

Ekman, S. 1954, Betrachtungen über die Fauna der abyssalen ozeanböden, Publ. Union intern.Sci.biol. 16(B):5-11.

Grassle, J.F. 1972, Species diversity, genetic variability and environmental uncertainty. In B. Battaglia (ed.), Fifth European Marine Biology Symposium, pp. 19-26, Piccin Editore, Padua.

Grassle, J.F. \& J.P. Grassle 1978, Life histories and genetic variation in marine invertebrates, In B. Battaglia and J. Beardmore (eds), Marine Organisms, pp.347-364, Plenum press.

Hansen, B. 1968, Brood-protection in a deep sea holothurian, Onei rophanta mutabilis Theel, Nature 217(5133):10621063.

Hansen, B. 1972, Photographic evidence of an unique type of walking in deep-sea holothurians, Deep Sea Res. 19:461-462.

Hansen, B. 1975, Systematics and biology of the deep sea holothurians, Galathea Rep. 13:1-262, 14 pls.

Hochachka, P.W. (ed) 1976, Biochemistry at depth. Pressure effects on biochemical systems of abyssal and midwater organisms. The 1973 Kona expedition of the Alpha Helix, Pergamon press Ltd:202 p.

Khripounoff, A. 1979, Relations trophiques dans l'écosystème benthique abyssal atlantique : Descriptions et bilan d'énergie, Thèse de 3ème cycle, Univ. Paris 6 .

Laubier, L. \& M. Sibuet 1977, Résultats des campagnes Biogas, 3 août 1972-2 no- 
vembre 1974, Res.Camp. à la Mer n 11 , 78 p., Publications CNEXO.

Laubier, L. \& M. Sibuet 1979, Ecology of the benthic communities of the deep North-East Atlantic, Ambio special report $6: 37-42$.

Lawrence, J.M. \& M. Sibuet 1979, Numbers and biomass of abyssal holothuroids (Echinodermata) from the Bay of Biscay, American Zoologist., 19:p.1010.

Lemche, H., B. Hansen, F.J. Madsen, O.S. Tendal \& T. Wolff 1976, Hadal life as analysed from photographs, Vidensk dansk naturb.Forer. 139:263-336.

Madsen, F.J. 1961, On the zoogeography and origin of the abyssal fauna in view of the knowledge of the Porcellanasteridae, Galathea rep. 4:177-218.

Mayr, E. 1974, Populations, espèces et évolution, Herman publ., $496 \mathrm{p}$.

Murphy, L.S., G.T. Rowe \& R. L. Haedrich 1976, Genetic variability in deep sea Echinoderms, Deep Sea Res. 23:339-348.

Pequegnat, W.E., B.M. James, A.H. Bouma, W.R. Bryant \& A.D. Fredericks 1972, Photographic study of Deep sea environments of the gulf of Mexico, Texas A \& M University oceanographic studies 3: 67-128, In contributions on the geological oceanography of the gulf of Mexico. V.J. Henry \& R. Rezak (ed.), Gulf Publ. Comp. Texas .

Rokop, F.J. 1975, Breeding patterns in the deep sea, Ph.D. University of California, San Diego, $174 \mathrm{p}$.

Roux, M. 1978, Ontogenèse et évolution des crinoïdes pédonculés depuis le trias, implications océanographiques, Thèse d'Etat, Université Paris XI, n 2082 : $1-167$.

Sanders, H. L. 1969, Benthic marine diversity and the stability time hypothesis, Brookhaven Symposia in Biology, 22: $71-81$.

Sanders, H.L. 1977, Evolutionary ecology and the deep sea benthos. The changing scenes in natural sciences 1776-1976, Academy of Natural Sciences, Special Pub1ic. 12:223-243.

Schoener, A. 1968, Evidence for reproductive periodicity in the deep sea, Ecology 49:81-87.

Sibuet, M. 1977, Répartition et diversité des Echinodermes (Holothurides, astérides) en zone profonde dans le golfe de Gascogne, Deep Sea Res. 24:549-563.

Smith, K.L. Jr 1978, Metabolism of the abysso pelagic rattail Coryphaenoides armatus measured in situ, Nature 274: 362-364.

Sokolova, M.N. 1958, Alimentation des invertébrés dẻtritivores en milieu abyssal (en russe), Trudy Inst. Oceanol., $27: 123-153$.
Sokolova, M.N. 1959, On the distribution of deep water bottom animals in relation to their feeding habits and the character of sedimentation, Deep Sea Res.,6:1-4.

Sokolova, M.N. 1972, Trophic structure of deep sea macrobenthos, Mar.Biol.,16(1): $1-12$.

Tyler, P.A. \& J.D. Gage, Reproduction and growth of the deep-sea brittlestar Ophiura 1 jungmani (Lyman), Oceano1.Acta 1980 (in press). 